\title{
LOKI - MOBILE LOCALIZATION AND WIKI-BASED INFORMATION FOR MUSEUMS
}

\author{
Peter Hoffmann \\ TZI Center for Computing and Communication Technologies, University Bremen, 28359 Bremen, Germany, \\ phoff@tzi.de
}

\begin{abstract}
In search of new target groups and in purpose to forge strong links to existing target groups museums try to use the support by technical features. Both, the internet for external communication and local technical infrastructures in the exhibitions, are used in that context. This paper introduces a research project which combines both aspects. An increasing number of museums present parts of their exhibitions in a digitized way as virtual exhibitions in the internet. Often information as well as interactive and entertaining elements are integrated in those presentations. In the physical ("real") exhibition the digitized information and especially interactive elements and presentation are used rarely. This situation gave the idea for the Loki-project which aims at a wiki-based application used in physical exhibitions.
\end{abstract}

Keywords: Social interaction, mobile media, wiki based information

\section{INTRODUCTION: THE IDEA OF A WIKISEUM}

Wiki systems are collections of web pages which are not only readable by the user but also editable online. So a wiki is an open content management system. For museums these kinds of systems might be interesting in several ways:

- A wiki system offers the opportunity to make objects and information easily accessible via the internet. This means a help for designing virtual exhibitions.

- A wiki system helps the user to become active. The (virtual) visitor has the chance to comment and discuss objects, to contribute personal knowledge, etc.

\subsection{THE WIKISEUM}

The media content of a museums' website is usually planned, prepared and produced in detail by a team of experts working at or for the museum. In most cases it is the content is static content (even if the media is a dynamic one like a video) and can only be watched by the user. The Wikiseum follows the approach to combine usual internet presentations of a museum with the wiki based functionalities like editing, deleting and linking information: the users have the chance to

- contribute their personal knowledge in the Wikiseum of the museum,

- integrate own media and information to it and

- take an active part in the design and the amount of the content.

This kind of activation may result in closing the gap between the museums' experts and researchers on the one hand and the visitors on the other hand as well as between the group of visitors, too.

As a first step this kind of activities are intended to increase the visitors' motivation to stay for a longer period of time on the website and to study the content in a more intensive way. As a second step this might lead to curiosity for the real exhibition and thus a visit of the real museum.

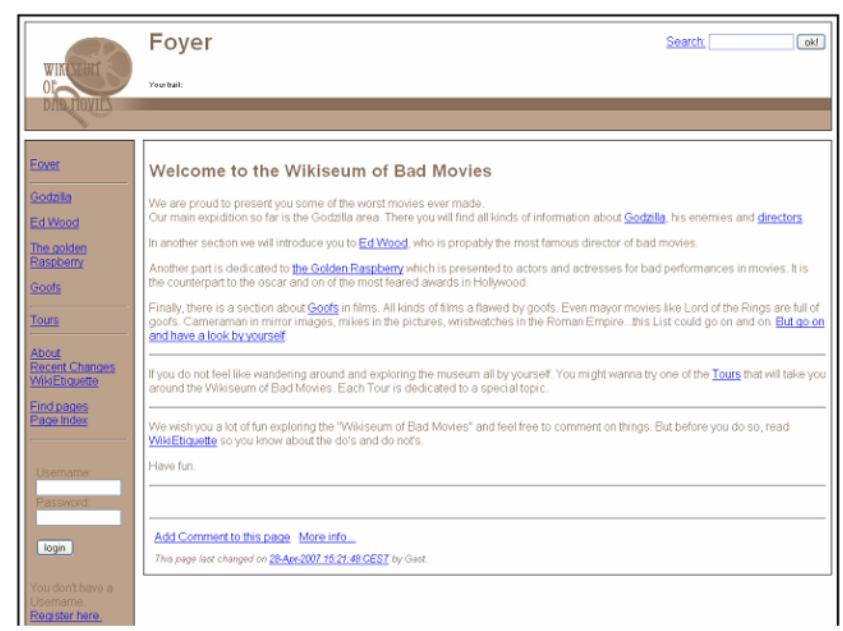

Fig. 1 - Home page of the "Wikiseum of Bad Movies" To give an impression that virtual exhibits, 
which meet the described ideas, are fundamentally feasible, the engine of the Wikiseum was developed [4]. It is based on an existing open wiki system, which was enhanced with some special features coming from the museums needs. A first virtual exhibition was realized with the topic of the "Wikiseum of Bad Movies" (Fig. 1).

\subsection{PROBLEMS OF SOCIAL AND INTELLECTUAL INTERACTION}

The development of the Wikiseum system and its realization and furthermore the time of running the system with the "Bad Movies" exhibition showed, that the offered freedom for the visitors to change the content and work with it offers several problems.

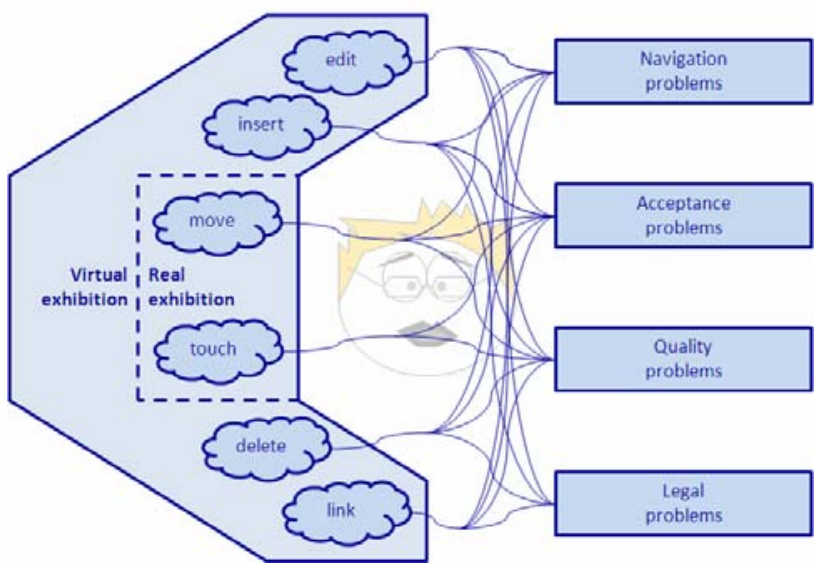

Fig. 2 - Problems in virtual and real exhibitions

In particular the imperative of "Do not touch", which is very often to be found in exhibitions, is opposed to the new kinds of freedom offered by the intellectual and social interaction of the system.

Like any other website, the content of cultural websites has to be planned, selected, produced and edited very carefully. Furthermore the content may not be changed by visitors to ensure correctness and form.

This is a contradiction to the usual wiki paradigm, in which every user can edit every part of the content. Such individual freedom in relation to the content implies problems for the provider:

- Legal problems because every user is able to add content, media and links, which leads e.g. to questions on copyrights.

- Quality problems because inserting and editing content may change quality and even the context of statements.

- Acceptability problems because the content becomes dynamic and may change over time. This might lead to incertitude for users who e.g. do not find things seen in the past.
Furthermore this dynamic behavior might have consequences for the quality of the content, which again reduces the acceptance.

- Navigation problems because of the dynamic characteristic of the content. E.g. links may appear and disappear so that well known paths through the virtual content may change and orientation is hindered.

These problems are stumbling blocks for cultural providers to realize parts of a website as a wiki system. This was the reason for developing a hierarchical role model for different types of users in the Wikiseum engine. Every single role has different rights for reading, writing and changing content (Fig. 3).

All users have access to the content: while unregistered users are only allowed to comment things and objects, registered users may add content and links. All entries of users have a limited lifetime after which the entry is deleted automatically if it is not "upgraded" by moderators or administrators.

Moderators and administrators are experts of the museum and responsible for the design and the content, including the control of the entries of any user.

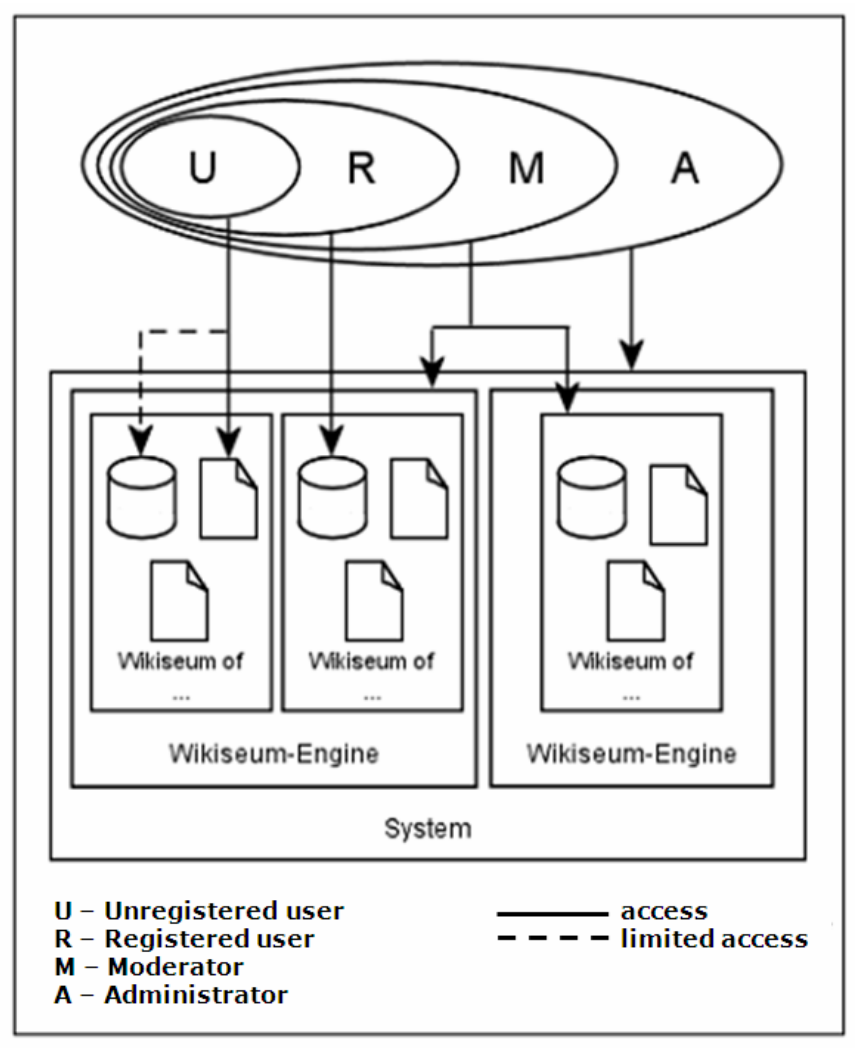

Fig. 3 - User roles in the Wikiseum (Hoffmann \& Herczeg) [3] 


\section{LOKI - COMBINATION OF REAL AND VIRTUAL LOCATIONS}

Under closer scrutiny a Wikiseum builds a separate second, virtual exhibition parallel to the real exhibition. The connection of both worlds has to be done on an abstract level by the user himself. Not until he decides to step into the virtual exhibition a connection between both information worlds can be build. But this needs a second step: the visited locations in the virtual exhibition have to be linked to the visited locations in the real exhibition.

\subsection{REAL AND VIRTUAL LOCATIONS}

The growth of the internet and its increasing usage seem to make it necessary to introduce a differentiation of real and virtual locations [6]. A physical location means a position or an object in the real world. It is mostly used as a reference point in the real environment. Since the growth of the internet however virtual locations get more and more important. These locations are mostly virtual meeting places like chat rooms or information sources in the web.

The combination of both virtual and real locations is attended by problems as it is difficult to develop virtual applications fitting to the real environment or to design information in an adequate way:

- Location based applications are mostly related to physical locations. Exceptions are augmented reality systems (AR-systems) and mobile gaming applications, where virtual locations are mapped to physical locations.

- While physical locations can be described by geometrical coordinates, virtual locations are described by the path to the information source or the web site.

- The presentation of information at virtual locations has to be realized in a completely different technical environment and under consideration of parameters of the cognitive psychology which are different in both kinds of locations. This often leads to a strict separation of both information worlds.

\subsection{SERVICES FOR REAL AND VIRTUAL LOCATIONS}

Location based services adapt themselves in the behavior and the presentation style to the location of the user. While in industrial contexts the term "location based service" is mostly used for mobile telecommunication services, in the field of computational research it is a subset of context sensitive services. As location based services offer a wide range of possible fields of application, all services of those kind try to offer automated access to information, which is adapted to the actual situational context.

The environmental situation of a location based service changes more or less constantly. To adapt the service to the changing parameters in an adequate way it has to be context sensitive. At least a physical motion might lead to a necessarily needed adaption of the service's behavior. So the position of the user is one of the decisive parameters [2]. But this is only one subset of the entire context [1], which is the combination of information about the state of the user, the state of the location or the object, and parameters which are relevant for the interaction between user and application or service. This kind of definition considers that the context to be aware of depends strictly of the kind of the service.

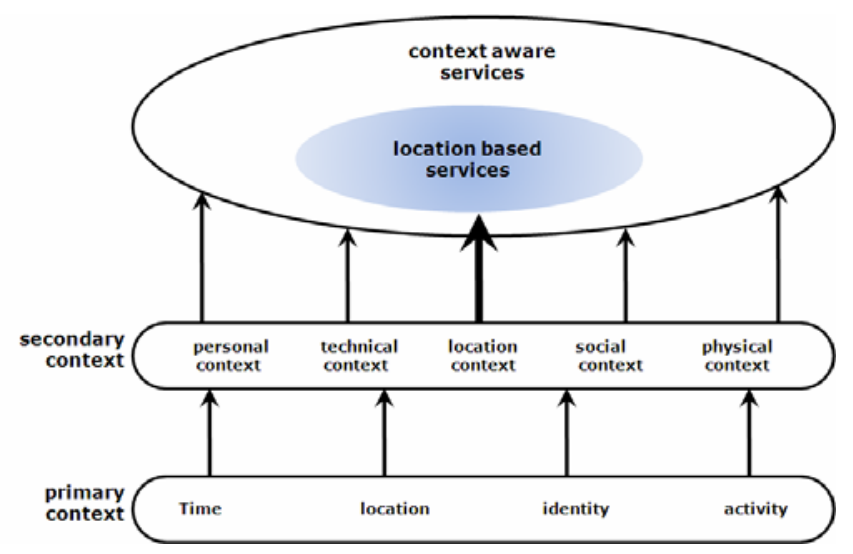

Fig. 4 - Context relation and location based service (see: Küpper) [6]

\subsection{THE LOKI APPROACH: COMBINATION OF REAL AND VIRTUAL LOCATIONS}

The Loki project takes the approach to avoid the separation of the information worlds by the use of context sensitivity as described in 2.1. For that purpose a situational context of the user is build, which connects his real location with relevant virtual locations. Information existing at the real location, which is in this case related to a single exhibit, is so linked to information existing in the virtual information world.

This combination aims for several advantages. All information stored, and especially the information stored in the virtual information world, can be used directly in the real exhibition. This feature is usually not realized yet in most exhibitions. Furthermore all kinds of elements for social and intellectual interaction typical for virtual information worlds can be integrated and used in the 
real information world, too. So advancing ones knowledge, taking part in discussions and comment locations are possible at any location.

Moreover if the user and his way through the information world, both real and virtual, is tracked, a profile of the user can be build which helps to give him suggestions and hints on the next possible steps on his way(s) according to his history and behavior.
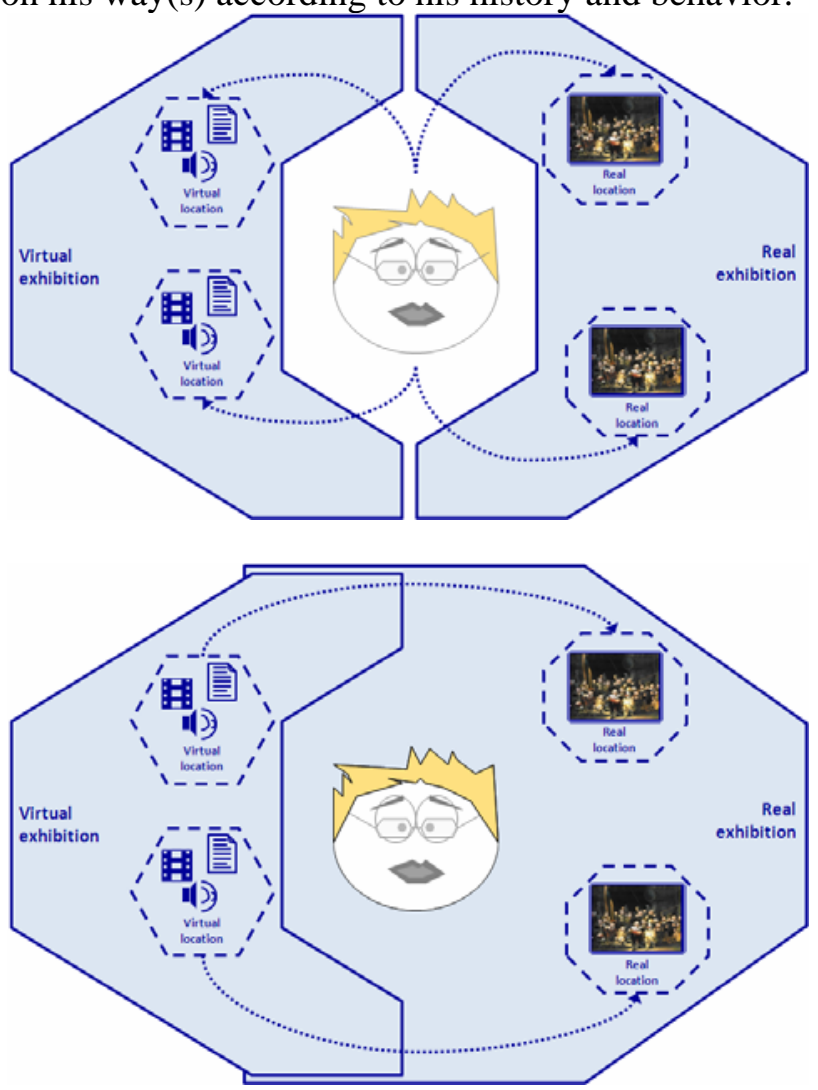

Fig. 5 - Real and virtual information worlds (top: separated; bottom: combined)

\section{THE PROTOTYPE: LOKI FOR THE "WIKISEUM OF BAD MOVIES"}

The prototype of Loki is developed as a clientserver-architecture. The server part consists of a Wikiseum, which itself is a further development of an existing open source wiki engine. It is a Java based implementation using Java Server Pages and Java Servlets for requests for presentation and editing. Both are embedded in a servlet container. The dynamic generation of the single web pages is activated via a web server.

The core part of the application is the demand for information related to a real location. The essential aspect is that posing a query is done automatically by the system and no interaction from the user is necessary. The actual position of the user in the real environment is detected by the application with RFID transponders. The prototype did not use dynamic, writable transponders but only readable ones. So the location related information or the path to it was not stored on the transponder. Only the transponder ID was read and used to start a request for the context information towards a database. The answer to this request consists in the name and a short description of the object, symbolic location descriptions and coordinates of the position in the real environment.

The latter are the basic references for the presentation of location based information and for calculating the ways to the next suggested locations to be visited. For an additional support for orientation the symbolic descriptions are used, which are drawn into the generated map. A symbolic description could so for example show the name or the number of the room, where an exhibit is shown.

The client for the Loki prototype was a PDA with a RFID reader. Both are used in combination to localize the user so that the presentation can be adapted. Moreover the PDA offers all input options the user needs during working with the application. These options are essentially necessary for the social and intellectual interaction. Other than in the related Dinharazade project [5], which was developed parallel to Loki and which uses audio interaction only, the interaction in Loki should take place generally as a text based interaction

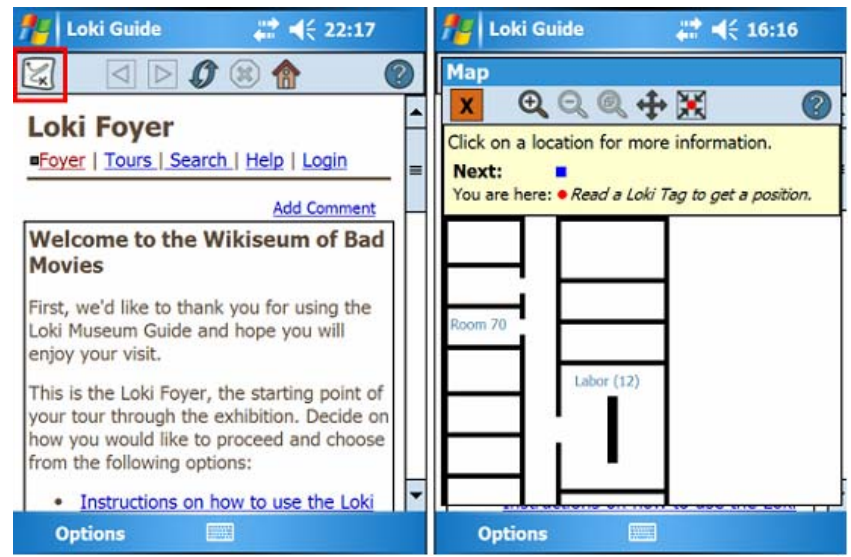

Fig. 6 - Web browser (left) and plan of the real museum (right) after starting the application

The Loki prototype was tested in an exhibition, which was specially therefore designed. An existing virtual exhibition, "The Wikiseum of Bad Movies" was the base to build up a real exhibition regarding to that topic.

The visitor starts the Loki application once at the beginning of his visit. The answer of the system is a starting screen similar to the entry of the "Wikiseum of Bad Movies" in the internet (Fig. 6 left) and an overview of the plan of the exhibition (Fig. 6 right). The user now has two options. First he can move around in the exhibition on his own ways. If he enters a location marked with a RFID transponder, the Loki application presents the related information 
stored in the Wikiseum on the PDA.

The second option for the user is to choose a guided tour through the (virtual) Wikiseum. In this option the focus of the Loki application is on the combination of both information worlds. The visitor gets hints from his Loki client where to go next on the tour. This means a walk to the next tour related location in the real exhibition. By entering this location the related information is presented and the way to the then following exhibit is determined.

Also during a guided tour the Loki system gives the user the freedom to leave the actual chosen tour e.g. for visiting other locations/ exhibits or for following links in the information world of the Wikiseum. Nevertheless the user is free to return to the chosen tour at any time. He only has to enter the last suggested location or he may use the "Back to the tour” Button of the application.

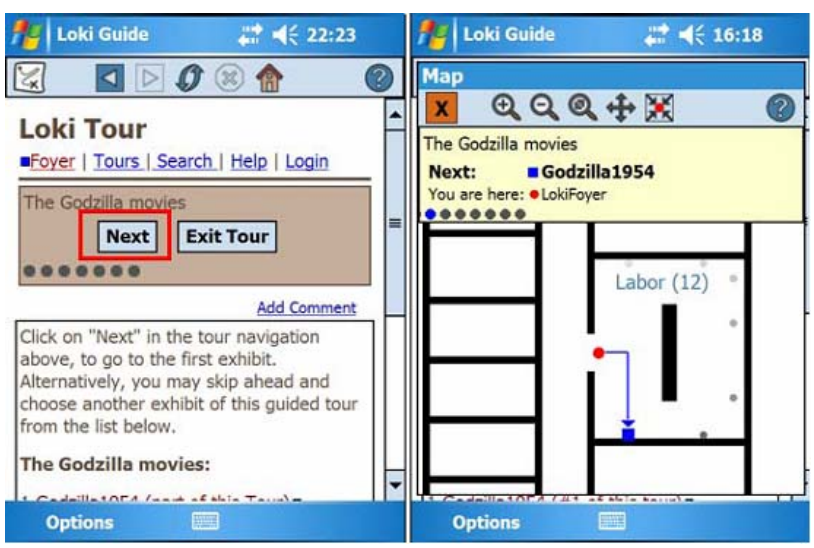

Fig.7 - Start page of the guided tour "The Godzilla Movies” (left) and path (right)

Independent of the chosen option the visitor can be socially and intellectually interactive at any time during his visit and during the use of the Loki application. He is allowed to comment any location and exhibit as well as any entry in the Wikiseum and to enrich the virtual exhibition with his personal knowledge. Besides this direct kind of interaction the ways, the user took through both, the real and the virtual exhibition, are tracked and registered if the user chose to allow the Loki client to do so. This option offers the user the possibility to access all seen information during his visit after he left the exhibition. So he can e.g. follow his own steps in the exhibition on his PC at home.

\section{EVALUATION}

The Loki project was evaluated with the main focus on the user guidance, which took place in two layers in parallel: in the real exhibition and in the virtual exhibition of the Wikiseum. Thus the most important factors of the evaluation were the choosing and the locating of the exhibits. Furthermore the acceptance of textual input under consideration of the PDA as a special device for this kind of task was another aim of the evaluation.

Those demands were the basis for the definition of user tasks like e.g. "Using the Loki client (in general)", "Registration and Log-In", "Locating a certain exhibit using the search function", "Choose and follow a guided tour" and "Comment a certain exhibit/entry". Moreover a list of formal principles had to be defined to analyze the application. The chosen criteria of the evaluation followed the principles of dialog design according to the german DIN standard DIN EN ISO 9241-110.

As expected the parallel navigation through two information worlds was a serious challenge for the users. Especially the option to follow a guided tour, but to be able to leave and re-enter it as well in the real as also in the virtual exhibition required an intensive examination and a long time of use of the system. Once overcome this obstacle the proactive characteristics of the system, realized in the automatic presentation of information without any explicit interaction by the user, was rated as a helpful assistance and an ease in the use of the Loki system.

Other problems coming from the social and intellectual interaction with the content can not be referred to the Loki application. Those problems came from the content itself, which was mainly text based media and so needed a way of textual interaction. This result of the evaluation is not a surprise as devices like PDAs are not really designed for extensive work with texts. Thus alternative ways of interaction should be found as remedy [5].

\section{OUTLOOK}

The Loki prototype can be developed into a final version from various aspects. So the use of other ways of localization could be supposed. So e.g. RFID-readers could be integrated into the exhibition environment stationary and the user is marked by a RFID transponder which is integrated in his ticket. In this contrary way of location the Loki server has to minute the user position and to send the information related to the determined location. For this approach it has to be considered that a more complex and larger infrastructure is necessarily needed. On the other hand it reduces the need to hand out a technical device to any visitor. From the design aspect has to be considered furthermore that one location may be related to more than one exhibit, which may cause in an unclear navigation through the virtual information world.

As a result during the development of the Loki prototype and its evaluation it became obvious that 
the biggest potential for development is to be found in the optimization of the interaction design. Audio input and output for the annotation as well as for the navigation through the virtual information world could offer a helpful alternative. If it is a benefit to use new kinds of devices with touch-based interaction or the like should be examined.

The next version of the Wikiseum and the Loki approach will be used in the TZI eXpo. This is an exhibition room run by the Center for Computing and Communication Technologies TZI at the University Bremen. In this exhibition scientific results, prototypes and demonstrators coming from R\&D projects of the TZI are displayed. The goal of combining the real TZI eXpo and its virtual counterpart is to strengthen the scientific communication by opening the actual scientific work to the broad public and make the researchers' work more transparent.

\section{REFERENCES}

[1] Dey A. K. \& Abowd G. D. Towards a Better Understanding of Context and ContextAwareness. GVU Technical Report GIT-GVU99-22, Georgie Institute of Technology, 1999.

[2] Dix A., Rodden T., Davies N., Trevor J., Friday A. \& Palfreyman K. Exploiting space and location as a design framework for interactive mobile systems. ACM Transactions on Computer-Human Interaction, Vol. 7, No. 3, Sept. 2000, pp. 285-312.

[3] Hoffmann P., Herczeg M. Wiki meets Museum - Die soziale Interaktion als Attraktivitätsgewinn für Web-Präsenzen im Kulturbetrieb. 1 . Österreichisches Wikiposium in Wien. In Stockinger, J., Leitner, H. (Hrsg.): Wikis im
Social Web. Wikiposium 2005/06, Österreichische Computer Gesellschaft, 2007.

[4] Hoffmann P., Herczeg M. Attraction by interaction: wiki webs as a way to increase the attractiveness of museums' Web sites. In; $J$. Trant and D. Bearman (eds.). Museums and the Web 2005: Proceedings, Toronto: Archives \& Museum Informatics. (Published March 31, 2005

at http://www.archimuse.com/mw2005/papers/hof fmann/hoffmann.html)

[5] Hoffmann P., Herczeg M. Soziale Interaktion mit Dinharazade - Kommentierung in einem interkativen mobilen Audiosystem in Museen und Ausstellungen. 2. Österreichisches Wikiposium. In Stockinger, J., Leitner, H. (Hrsg.): Wikis im Social Web. Wikiposium 2005/06, Österreichische Computer Gesellschaft, 2007.

[6] Küpper A. Location-Based Services Fundamentals and Operations. Chichester: John Wiley \& Sons, 2005.

Peter Hoffmann, born 1965; 1990 - 2000 Studies of Safety Engineering (University Wuppertal, Germany); 2000 - 2002 Scientific researcher (Computer Graphics Center ZGDV, Darmstadt, Germany); 2002 - 2008 Scientific researcher (University of Luebeck, Germany; Institute for Multimedia and Interactive Systems); since 2008 Scientific researcher and managing director (University Bremen, Germany, TZI Center for Computing and Communication Technologies). 